\title{
PRO-QUEST: A rapid assessment method based on PROgressive saturation for Quantifying Exchange Rates using Saturation Times in Chemical Exchange Saturation Transfer (CEST)
}

Eleni Demetriou ${ }^{1,{ }^{*}}$, Mohamed Tachrount ${ }^{1}$, Moritz Zaiss ${ }^{2}$, Karin Shmueli ${ }^{3}$, Xavier Golay ${ }^{1}$

${ }^{1}$ Brain Repair \& Rehabilitation, Institute of Neurology, University College London, UK

${ }^{2}$ High Field Magnetic Resonance, Max Planck Institute for Biological Cybernetics, Tübingen, Germany

${ }^{3}$ Department of Medical Physics and Biomedical Engineering, University College London, UK

Word count: 6240

I. I. Rabi Young Investigator Award Competition

Submitted to Magnetic Resonance in Medicine

${ }^{*}$ Correspondance to:

Eleni Demetriou

Institute of Neurology

Queen Square

University College London

London WC1N 3BG

United Kingdom

Running Title: PRO-QUEST: A rapid method for CEST exchange rate quantification 
PRO-QUEST: A rapid method for CEST exchange rate quantification

\section{Abstract}

Purpose: To develop a new MRI technique to rapidly measure exchange rates in Chemical Exchange Saturation Transfer (CEST) MRI.

Theory and Methods: A novel pulse sequence for measuring chemical exchange rates through a progressive saturation recovery process, called PRO-QUEST (PROgressive saturation for Quantifying Exchange rates using Saturation Times), has been developed. Using this method, the water magnetization is sampled under non-steady state conditions and off-resonance saturation is interleaved with the acquisition of images obtained through a Look-Locker type of acquisition. A complete theoretical framework has been set up, and simple equations to obtain the exchange rates have been derived.

Results: A reduction of scan time from 58 to $16 \mathrm{~min}$ has been obtained using PROQUEST vs. the standard QUEST. Maps of both longitudinal relaxation time of water $\left(T_{1}\right)$ and radio frequency pulse amplitude $\left(B_{1}\right)$ can simply be obtained by repetition of the sequence without off-resonance saturation pulses. Simulations and calculated exchange rates from experimental data using amino-acids such as glutamate, glutamine, taurine and alanine were compared and found to be in good agreement. The PRO-QUEST sequence was also applied on healthy and infarcted rats after 24 hours and revealed that imaging specificity to ischemic acidification during stroke was substantially increased relative to standard APT-weighted imaging.

Conclusion: Because of the reduced scan time and insensitivity to non-chemical exchange factors such as direct water saturation, PRO-QUEST can serve as an excellent alternative for researchers and clinicians interested to map $\mathrm{pH}$ changes in vivo. 
PRO-QUEST: A rapid method for CEST exchange rate quantification

\section{Introduction}

Chemical Exchange Saturation Transfer (CEST) magnetic resonance imaging (MRI) is an emerging method for detecting signals from low concentrated species in vivo and for monitoring changes in environmental parameters such as $\mathrm{pH}$, temperature, and ion concentration [1]. The chemical exchange rate has been used as a quantitative imaging biomarker for $\mathrm{pH}$ mapping or to guide selection of the most appropriate CEST MRI parameters. Indeed, the chemical exchange of protons within exchangeable groups such as amides and amines found in vivo is base-catalyzed, and will therefore vary with $\mathrm{pH}$ [2],[3]. When combined with other techniques such as diffusion-weighted imaging (DWI) or perfusion-weighted imaging (PWI), $\mathrm{pH}$ maps have shown great potential for improving our understanding of acute ischemic damage or for monitoring cancer progression and its response to various treatments [4], [5].

Existing methods for quantitative measurements of soluble labile proton exchange rates in water are either spectroscopy-based, or rely on multiple repetitions of the same acquisition following changes in the experimental parameters [5],[6],[7]. With nuclear magnetic resonance (NMR) spectroscopy, the exchange rates of protons in the slow exchange regime can be estimated from their linewidths measured on solute molecules [5]. Water exchange spectroscopy (WEX) has been used to assess the exchange properties of amides in endogenous mobile proteins and peptides in the brain [6]. Through the build-up of the signal from the exchangeable protons as a function of mixing time the exchange rate can be calculated [6],[8],[9]. Although slow exchange rates are perfectly quantifiable, fast exchanging species require very short mixing times which are difficult to attain due to limitations in hardware, especially in clinical MRI systems.

Recently, measurements of exchange rates using imaging techniques have been developed to overcome the limited spatial resolution of MRS measurements and to provide quantitative maps in both healthy and diseased states. Exchange rates can be quantified as a function of saturation time using Quantification of Exchange rates using Saturation Times (QUEST) or Saturation Power (QUESP) [5],[10]. Moreover, in the case of continuous saturation, analytical solutions of the Bloch-McConnell (BM) 
PRO-QUEST: A rapid method for CEST exchange rate quantification

equations have been derived to provide fast and accurate estimates of the exchange rates [11]. Other methods of quantification include the omega plot method, working without a priori knowledge of the agent concentration; or the extended QUEST method with ratiometric analysis (QUESTRA), which considers the rate at which the label and reference scans approach the steady state [12].

While different methods have been proposed to enable correct estimates of the exchange rates, they all have in common that they require too much time for their utility to really be explored in the clinic. To eliminate the need for long CEST saturation times and long TR, MRI fingerprinting methods have also been used for simultaneous multiparametric mapping to quantify exchange rates [13-15]. Advantages of this approach include sensitivity, specificity and speed [14]. However, even in relatively simple phantom studies, quantification of exchange rates or concentrations can go wrong due to relatively poor fingerprinting trajectory efficiency [14]. In addition, quantification of exchange rates in vivo is very difficult due to the interplay of multiple molecular processes between e.g. healthy volunteers and patients.

Here, we propose a novel pulse sequence for measuring chemical exchange rates through a progressive saturation recovery process, called PRO-QUEST for PROgressive saturation for Quantifying Exchange rates using Saturation Times in CEST. This technique aims to provide direct measures of chemical exchange rates, while at the same time dramatically reducing the total scan time required. It works based on the water magnetization being sampled along a Look-Locker sampling scheme, thereby providing maps of $\mathrm{T}_{1}$ and $\mathrm{B}_{1}$, both needed for exchange-rate calculations. Measurements of exchange rates are obtained in phantoms of various amino acids and in rat brains. In addition, PRO-QUEST measurements made in phantoms are compared with those made using the traditional QUEST method, and the sensitivity of the technique to various parameters is explored. 
PRO-QUEST: A rapid method for CEST exchange rate quantification

\section{Theory}

\section{Progressive saturation $\mathrm{T}_{1}$ mapping}

Fast measurement of longitudinal-relaxation times $\left(T_{1}\right)$ can be performed using a single shot method known as the Look-Locker technique [16]. It is typically based on the use of an inversion or a saturation pulse, followed by multiple small flip-angle pulses, which sample the recovery of the longitudinal magnetization (see Figure 1). The signal evolution during a Look-Locker experiment for a number (n) of RF pulses of the same

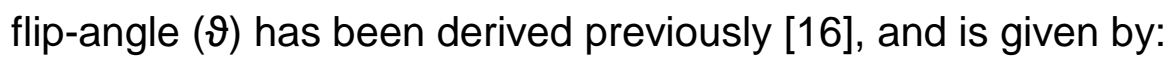

$M_{z d}(n \tau)=\frac{1-\left[(\cos \vartheta)^{n-1} e^{-(n-1)\left(\tau R_{1}\right)}\right]}{1-\left[(\cos \vartheta) e^{-\left(\tau R_{1}\right)}\right]} M_{z d}(\tau)+M_{\mathrm{eq}}\left(1-e^{-\left(t_{d} R_{1}\right)}\right)\left[(\cos \vartheta)^{n-1} e^{-(n-1)\left(\tau R_{1}\right)}\right]$

where $M_{z d}(\tau)=M e q\left(1-e^{-\left(\tau R_{1}\right)}\right)$ in the case of a saturation-recovery Look-Locker experiment, $t_{d}$ is the time to first excitation from preparation, $\mathrm{n}$ is the number of excitation pulses, $\tau$ is the time between $\vartheta$ pulses, $R_{1}=1 / T_{1}$ with $T_{1}$ being the water longitudinal relaxation time and $M_{e q}$ is the equilibrium magnetization.

Both $\mathrm{T}_{1}$ and $M_{e q}$ can be estimated using Equation 1. In practice, an appropriate range of flip angles $\vartheta$, no larger than $20^{\circ}$, has to be selected so that the small flip angle approximation holds and the measured $T_{1}$ does not deviate from its theoretical value [17], [18] . By using an optimal set of sampling parameters (i.e. $n, \vartheta$ and $\tau$ ), $T_{1}$ can be measured with good accuracy and in a reasonable imaging time [18].

\section{Exchange rate measurements through progressive saturation recovery}

PRO-QUEST was implemented by inserting either an off-resonance saturation module consisting of a single RF pulse or a spin-lock module before each readout module of the Look-Locker method so that the saturation effects progressively accumulate, while the measured signal is recovering under the longitudinal relaxation. To get accurate estimates of exchange rates, PRO-QUEST is applied with and without saturation module as depicted in Figure 1. The saturation pulse parameters are: pulse width (tsat), flip angle (fa) and number of pulses (n). Imaging parameters for the readout are:

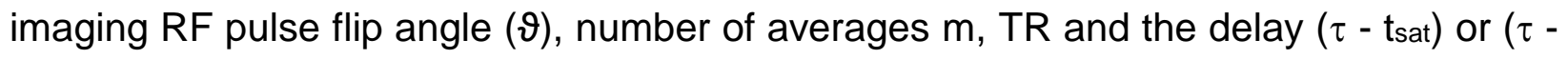


PRO-QUEST: A rapid method for CEST exchange rate quantification

tdel) between the readout and the start of the following acquisition $\vartheta$ pulse. Generally, the saturation module used in this method is centered at the offset frequency of interest e.g. amine, amide or hydroxyl frequency offsets ( 1 - 4 ppm from water), but the module can be extended beyond a single saturation frequency or a spin lock pulse can also be used on resonance for very rapidly exchanging groups resonating close to the water line (e.g. hydroxyl protons).

Mathematical equations have been derived to describe the recovery curves in the presence of CEST saturation pulses [11],[19]. In general, one of the main characteristics of the signal evolution during a CEST off-resonance pulse is that the (apparent) relaxation rate evolves with $\mathrm{R}_{1 \rho}$ (the relaxation rate along the effective field) rather than $\mathrm{R}_{1}$. Combining this fact with Equation 1, the longitudinal water magnetization after $\mathrm{n}$ off-resonance saturation pulses $\left(M_{z s a t}\right)$ can be described as (see Appendix for full derivation):

$$
\begin{aligned}
& M_{z s a t}(n \tau)=\frac{1-\left[(\cos \vartheta)^{n} e^{-n\left(\tau R_{1}-t_{s a t}\left(R_{1}-R_{1} \rho\right)\right)}\right]}{1-\left[(\cos \vartheta) e^{-\left(\tau R_{1}-t_{s a t}\left(R_{1}-R_{1} \rho\right)\right.}\right]} M_{z s a t}(\tau) \\
& \quad+M_{\text {eq }}\left(1-e^{-\left(t_{d} R_{1}\right)}\right)\left[(\cos \vartheta)^{n} e^{-n\left(\tau R_{1}-t_{s a t}\left(R_{1}-R_{1} \rho\right)\right)}\right] \\
& \text { where } M_{\text {zsat }}(\tau)=M_{\text {ss }}\left(1-e^{-\left(R_{1} \rho t_{s a t}\right)}\right) \cos \vartheta e^{-\left(\left(\tau-t_{\text {sat }}\right) R_{1}\right)}+M_{\text {eq }}\left(1-e^{-\left(\left(\tau-t_{s a t}\right) R_{1}\right)}\right)
\end{aligned}
$$

and $M_{S S}=\frac{R_{1} \cos ^{2} \varphi}{R_{1 \rho}}$ is the steady state magnetization, $t_{s a t}$ is the duration of the CEST offresonance saturation pulse, $\cos \varphi=\frac{\Omega}{\sqrt{\omega_{1}^{2}+\Omega^{2}}}$, with $\varphi$ is the angle between the effective field and the z-axis, $\Omega$ is the frequency offset with respect to water (i.e $\omega_{\mathrm{rf}}$ ) and $\omega_{1}=\gamma \mathrm{B}_{1}$ is the amplitude of the RF field.

For a continuous-wave irradiation, and assuming that the spin relaxation is dominated by a single exponential decay the relaxation constant along the effective field, $R_{1} \rho$ can generally be expressed as [19] :

$R_{1 \rho}=R_{1}(\cos \varphi)^{2}+\left(R_{2}+R_{e x}\right)(\sin \varphi)^{2}$ 
PRO-QUEST: A rapid method for CEST exchange rate quantification

$R_{e x}=\frac{\rho_{B} \delta^{2} k_{e x}}{\left((\delta-\Omega)^{2}+\omega_{1}^{2}+k_{e x}^{2}\right)}$ is the exchange-dependent relaxation which induces the CEST effect, and $R_{1}$ and $R_{2}$ the longitudinal and transverse relaxation of water in the absence of chemical exchange. Here, $\rho_{B}$ is the fractional concentration of the labile exchangeable protons, $k_{e x}$ is the exchange rate from the exchangeable protons to water, and $\delta$ is the Larmor frequency of the exchangeable labile protons.

For on-resonance spin-locking (SL) $\left(\varphi=90^{\circ}\right), R_{1 \rho}=R_{2}+R_{e x}$ while for off-resonance SL (where $\left.\varphi=\arctan \left(\frac{\omega_{1}}{\Omega}\right)\right)$ :

$R_{1 \rho}=R_{1} \frac{\Omega^{2}}{\Omega^{2}+\omega_{1}^{2}}+\left(R_{2}+R_{e x}\right) \frac{\omega_{1}^{2}}{\Omega^{2}+\omega_{1}^{2}}=R_{1}+\left(R_{2}-R_{1}\right) \frac{\omega_{1}^{2}}{\Omega^{2}+\omega_{1}^{2}}+R_{e x} \frac{\omega_{1}^{2}}{\Omega^{2}+\omega_{1}^{2}}$

The exchange-dependent relaxation term for an offset $\Omega$ can be written as [11]:

$R^{\prime}{ }_{e x}(\Omega)=R_{e x}(\sin \varphi)^{2}=\frac{\rho_{B} \delta^{2} k_{e x}}{\left((\delta-\Omega)^{2}+\omega_{1}^{2}+k_{e x}^{2}\right)} \frac{\omega_{1}^{2}}{\Omega^{2}+\omega_{1}^{2}}$

$R^{\prime}{ }_{\text {ex }}$ reaches a peak at $\Omega=\delta$ (i.e. on-resonance with the CEST peak) and assuming that $\delta \gg \omega_{1}$ it can be simplified to:

$R^{\prime}{ }_{e x}(\delta)=\rho_{B} k_{e x} \frac{\omega_{1}^{2}}{k_{e x}^{2}+\omega_{1}^{2}} \frac{\delta^{2}}{\delta^{2}+\omega_{1}^{2}}=\rho_{B} k_{e x} \frac{\omega_{1}^{2}}{k_{e x}^{2}+\omega_{1}^{2}}$

Therefore, for $\delta \gg \omega_{1}$

$R_{1 \rho}(\delta)=R_{1}+\left(R_{2}-R_{1}\right) \frac{\omega_{1}^{2}}{\delta^{2}+\omega_{1}^{2}}+\rho_{B} k_{e x} \frac{\omega_{1}^{2}}{k_{e x}^{2}+\omega_{1}^{2}}$

For off-resonance spin lock or off-resonance continuous wave irradiation, Equation 7 can be applied to deduce the exchange-dependent relaxation term $R^{\prime}{ }_{e x}$ as a function of $\omega_{1}=\gamma B_{1}$. However, for off-resonance shaped RF pulses, it was shown previously [20] that $\bar{R}_{1 \rho}$ yields better estimates of exchange rates and fractional concentrations rather than using $R_{1 \rho}\left(\overline{\omega_{1}(t)}\right) \cdot \bar{R}_{1 \rho}$ is defined as [20]:

$$
\begin{aligned}
& \bar{R}_{1 \rho}=\frac{1}{t_{\text {sat }}} \int_{0}^{t_{\text {sat }}} R_{1 \rho}(t) d t=R_{1}+\frac{1}{t_{\text {sat }}} \int_{0}^{t_{\text {sat }}}\left(R_{2}-R_{1}\right) \frac{\omega_{1}^{2}(t)}{\delta^{2}+\omega_{1}^{2}(t)} d t+\frac{1}{t_{\text {sat }}} \int_{0}^{t_{\text {sat }}} \rho_{B} k_{\text {ex }} \frac{\omega_{1}^{2}(t)}{k_{\text {ex }}^{2}+\omega_{1}^{2}(t)} d t \\
& =\overline{R_{\text {eff }}}+\overline{R_{\text {ex }}^{\prime}} \text { (8) }
\end{aligned}
$$


PRO-QUEST: A rapid method for CEST exchange rate quantification

where $t_{s a t}$ is the length of the off-resonance CEST saturation pulse.

To estimate exchange rates, $T_{1}, B_{1}$ and $M_{\text {eq }}$ maps are calculated by fitting Equation 1 to the signal evolution over time, obtained with the pulse sequence displayed in Figure 1 (b), similarly with previous work [21]. The obtained $T_{1}, \vartheta$ and $M_{e q}$ are then used as input parameters for estimating the exchange-induced relaxation term $\overline{R_{e x}^{\prime}}$ or the exchange rates $\left(k_{e x}\right)$ by fitting the progressive saturation recovery curves as a function of number of saturation pulses to Equation 2 in which $R_{1 \rho}$ is substituted with $\bar{R}_{1 \rho}$, as shown in Equation 8, to obtain an estimate of $\overline{R^{\prime}}$ ex . This is then used in conjunction with $\rho_{B}$ and the integral $\int_{0}^{t_{s a t}} \frac{\omega_{1}^{2}(t)}{k_{e x}^{2}+\omega_{1}^{2}(t)} d t$ which is solved numerically to estimate the exchange rates $\left(k_{e x}\right)$ (see Equation 8). In this work, $\rho_{B}$ is provided to the fit or data are acquired at two irradiation amplitudes $\omega_{1,2}$ and the concentration-free $\overline{R_{e x}^{\prime}}$ is calculated as $\overline{R_{\text {ex }}^{\prime}}\left(\omega_{1}\right) / \overline{R_{\text {ex }}^{\prime}}\left(\omega_{2}\right)$, which can be then used to extract the exchange rate numerically. The minimum set of measurements to allow calculation of an exchange rate $k_{e x}$ is:

- Measure $T_{1}, B_{1}$ and $M_{e q}$ using the pulse sequence displayed in Figure 1(b) at two different flip angles $\vartheta_{1}$ and $\vartheta_{2}$

- Measure $\overline{R_{e x}^{\prime}}$ using the pulse sequence displayed in Figure 1(a) by applying the CEST off-resonance saturation pulses at two irradiation amplitudes $\omega_{1}$ (e.g. $\omega_{1}$ and $\left.2 \omega_{1}\right)$

- Measure $T_{2}$ using a Carr-Purcell-Meiboom-Gill (CPMG) sequence at different echo times. Even echoes are fitted using a mono-exponential signal decay to obtain $\mathrm{T}_{2}$. 
PRO-QUEST: A rapid method for CEST exchange rate quantification

\section{Methods}

Several phantom experiments using amino acid samples at different $\mathrm{pH}$ values were made to test the theory. QUEST/QUESP experiments were performed in the same samples to validate the exchange rates obtained with PRO-QUEST. $\mathrm{pH}$ and solute concentrations were varied to investigate the dependence of the measured exchange rates on various parameters.

PRO-QUEST experiments were also performed in 3 healthy and 3 infarcted rats to demonstrate the feasibility of obtaining exchange rate measurements in vivo and their potential usefulness in a clinical setting.

$\mathrm{T}_{2}$ values, necessary for both PRO-QUEST and QUEST/QUESP methods, were acquired in both phantoms and rats using a standard CPMG experiment.

Precision of $T_{1}$ measurements from PRO-QUEST was compared to gold-standard methods using an inversion-recovery spin-echo echo-planar-imaging sequence in both phantoms and healthy rats.

Estimation of $B_{1}$ from PRO-QUEST was obtained in phantoms and healthy rats at three imaging flip angles (i.e. $8^{\circ}, 15^{\circ}, 25^{\circ}$ ).

\section{Phantom solutions}

Taurine, alanine, glutamate and glutamine possess amine protons which are all found to resonate close to 3.00ppm (Gln: 2.87ppm; Glu: 3.00ppm; Tau: 2.87ppm; Ala: 2.87ppm) and produce substantial CEST contrast (see Supporting Figure S1). These metabolites were chosen to assess whether the overlapping CEST signals at $3.00 \mathrm{ppm}$ could be discriminated based on their different exchange properties.

All samples were prepared using $0.1 \%$ phosphate-buffer-saline (PBS) buffer and scanned within the same day to avoid potential pH drifts (i.e. the gradual absorption of carbon dioxide from the atmosphere leads to an increase in $\left[\mathrm{H}^{+1}\right]$ which decreases the $\mathrm{pH})$. Exchange rate measurements were obtained from $100 \mathrm{mM}$ taurine, alanine, 
PRO-QUEST: A rapid method for CEST exchange rate quantification

glutamine and glutamate samples prepared at various $\mathrm{pH}$ values ranging between 5.8 and 7.4 .

Additional experiments were performed by varying the solute concentration from 12.5 $\mathrm{mM}$ to $100 \mathrm{mM}$ under constant $\mathrm{pH}=6.2$ for assessing the dependence of the PROQUEST curves on the concentration of solute. For all the experiments, the $\mathrm{pH}$ was measured using a micro pH probe (Mettle-Toledo International, Inc.) and adjusted where necessary with the use of sodium hydroxide $(\mathrm{NaOH})$ and hydrochloric acid $(\mathrm{HCl})$. Syringes of $1 \mathrm{ml}$ volume capacity were used as phantom containers, sealed with silicon glue. All phantoms were grouped in sets of five (same concentration, and five different $\mathrm{pH}$ values) or group of four (same $\mathrm{pH}$, and different concentrations). The temperature was kept constant at $23^{\circ} \mathrm{C}$ throughout the experiment.

\section{In vivo experiments}

All animal experiments were performed in accordance with the UK Animals (Scientific Procedures) Act 1986, and the Directive 2010/63/EU of the European Parliament and of the Council on the Protection of Animals Used for Scientific Purposes. Six SpragueDawley (SD) rats (weighing 200-250g) were used for MRI experiments. Prior to each scan, animals were anaesthetized with isoflurane. A concentration of $3 \%$ isoflurane in air was used for induction of anesthesia, and then the animals were transferred to the scanner bed and a facemask was applied to ensure continuous supply of anesthetic. Isoflurane concentration was decreased to $1.5-2.0 \%$ and maintained at this level for the rest of the experiment. During the scanning, the body temperature was maintained at $37^{\circ} \mathrm{C}$ with a heating pad, while respiration rate was constantly monitored using MR compatible devices (Small Animal Instruments, Inc. Stony Brook, NY, USA).

\section{Stroke procedure}

Three male SD rats (weighing 200-250g) were anaesthetized with isofluorane (3\% for induction and $2 \%$ during surgery) via a vaporizer with a mixture of air. A middle cerebral artery occlusion was performed as follows [22]. A midline neck incision was made over 
PRO-QUEST: A rapid method for CEST exchange rate quantification

the trachea and the tissues were retracted gently. The common carotid artery (CCA), internal carotid artery (ICA) and external carotid artery (ECA) were isolated and the CCA and ECA ligated. A filament was inserted from the ECA into the ICA to occlude the origin of the middle cerebral artery. After an ischemic time of $45 \mathrm{~min}$, the area was cleaned and rehydrated with saline. Finally, the skin was sutured and analgesics such as Rimadyl and Ketamine were given post operatively. The animals were kept in a warm environment during recovery after MCA occlusion. All the animals were scanned at baseline and at 24 hours post stroke.

\section{Pulse sequence}

\section{Phantom experiments}

The PRO-QUEST sequence was implemented on a 9.4 Tesla Agilent MRI scanner. A detailed diagram of the pulse sequence is displayed in Figure 1. Using the sequence in Figure $1(a)$, each of $n=300$ off-resonance saturation pulses (asymmetric sinc-Gauss) was centered at $3.0 \mathrm{ppm}$ with parameters: $\mathrm{t}_{\mathrm{sat}}=6.77 \mathrm{~ms}$, fa $=90^{\circ}, 180^{\circ}, 300^{\circ}, 400^{\circ}, 500^{\circ}$, $700^{\circ}, 900^{\circ}$ (corresponding to $\mathrm{B}_{1}=0.87 \mu \mathrm{T}, 1.74 \mu \mathrm{T}, 2.89 \mu \mathrm{T}, 3.85 \mu \mathrm{T}, 4.82 \mu \mathrm{T}, 6.75 \mu \mathrm{T}$ and $8.67 \mu \mathrm{T}$, with $\left.\mathrm{B}_{1}=\mathrm{fa} / \mathrm{yt}_{\mathrm{sat}}\right)$ and bandwidth $=1000 \mathrm{~Hz}(2.5 \mathrm{ppm})$. Multiple flip angles were used to investigate the dependence of $\overline{R_{e x}^{\prime}}$ on the irradiation amplitude. However, in principle, two $B_{1}$ values are enough to remove the concentration dependency in Equation 8 and provide estimates of the exchange rates. To estimate $T_{1}, B_{1}$ and $M_{e q}$, an additional scan was acquired without saturation pulses (Figure 1(b)). This was also repeated with flip angles $\vartheta=8^{\circ}, 15^{\circ}$ and $25^{\circ}$ to allow estimation of $B_{1}$ and the error in $T_{1}$ due to variations in $\vartheta$.

A single-slice 2D-GRE sequence with FOV $20 \times 20 \mathrm{~mm}^{2}$, data matrix $64 \times 64, T R=2.6 \mathrm{~s}$, $\mathrm{TE}=1.3 \mathrm{~ms}$, slice thickness $=4 \mathrm{~mm}$ was employed for readout. PRO-QUEST measurements were made in phantoms using a transmit/receive $33 \mathrm{~mm}$ RF coil (all RF coils: Rapid Biomedical, Germany). The total scan time for a single PRO-QUEST exchange rate measurement was $28 \mathrm{~min}$ when 7 irradiation powers are used ( $2.8 \mathrm{~min}$ per acquisition) and can be further reduced to $14 \mathrm{~min}$ for 2 irradiation powers.

\section{In vivo experiments}


PRO-QUEST: A rapid method for CEST exchange rate quantification

Three healthy and three infarcted SD rats were scanned using a $72 \mathrm{~mm}$ volume coil for radio frequency $(\mathrm{RF})$ transmission and a two-element receive array coil. The optimized sequence consisted of two adiabatic half-passage saturation pulses for improved saturation [23] , followed by 150 off-resonance saturation pulses centered at 3.5ppm with RF pulse parameters: $t_{s a t}=16.93 \mathrm{~ms}, f a=90^{\circ}$ and $180^{\circ}$ and bandwidth $=400 \mathrm{~Hz}(1$ ppm). This off-resonance saturation frequency was chosen to assess the changes in amide proton exchange following ischemia as demonstrated earlier [4]. Images were collected with slice thickness $=2 \mathrm{~mm}$, FOV $=35 \times 35 \mathrm{~mm}^{2}$, matrix size $=64 \times 64$, TR=3.0s, $\mathrm{TE}=1.12 \mathrm{~ms}$, and $\vartheta=8^{\circ}$. For exchange rate measurements using PRO-QUEST, $\mathrm{T}_{1}, \mathrm{~B}_{1}$ and $M_{e q}$ were calculated using the multiple flip angle strategy described above. The same PRO-QUEST MRI protocol was employed for the stroke study with exchangeweighted images collected at $\mathrm{fa}=90^{\circ}$ and $180^{\circ}\left(0.35 \mu \mathrm{T}\right.$ and $0.69 \mu \mathrm{T}$ B $\mathrm{B}_{1}$ respectively). Two irradiation powers were used for concentration-free $\overline{R_{e x}^{\prime}}$, which was calculated as $\overline{R^{\prime}{ }_{e x}}(0.35 \mu \mathrm{T}) / \overline{R^{\prime}{ }_{e x}}(0.69 \mu \mathrm{T})$. It can be seen from Equations 5 and 6 that when we divide the $\overline{R_{e x}^{\prime}}$ obtained at two different irradiation amplitudes the result will be unaffected by any changes in the concentration of exchangeable protons $\rho_{B}$. The total scan time was $16 \mathrm{~min}$ ( $3.2 \mathrm{~min}$ per acquisition).

\section{QUEST/QUESP MRI}

QUEST measurements were acquired in the same samples described above using a single-shot spin-echo (SE) echo planar imaging (EPI), ( $T R=65.3 \mathrm{~ms}, T E=4.07 \mathrm{~ms}$, $\mathrm{FOV}=20 \times 20 \mathrm{~mm}^{2}$, slice thickness $=5 \mathrm{~mm}$, matrix size $=64 \times 64$ ) with a saturation train prior to the readout consisting of a saturation pulse train of $1,21,41,61,101$ and 151 Gaussian pulses at two different RF amplitudes: $1.14 \mu \mathrm{T}$ and $1.8 \mu \mathrm{T}$ ( $\mathrm{t}_{\mathrm{sat}}=50 \mathrm{~ms}, \mathrm{fa}=900^{\circ}$ and $1400^{\circ}, 91 \%$ duty cycle). $B_{1}$ was calculated as the mean $B_{1}$ integrated over an RF pulse and is equal to $B_{1}=f a / y t_{s a t}$. The total scan time needed for a single QUEST exchange rate measurement was $58 \mathrm{~min}$ (9.7min per acquisition with different irradiation lengths) and additional $14 \mathrm{~min}$ were needed for obtaining a $T_{1}$ map, leading to a total acquisition time of $1 \mathrm{~h}$ and $12 \mathrm{~min}$. 
PRO-QUEST: A rapid method for CEST exchange rate quantification

QUESP measurements were also acquired in the same samples using 151 Gaussian pulses at eleven different irradiation amplitudes (i.e. $0.39 \mu \mathrm{T}, 0.76 \mu \mathrm{T}, 1.17 \mu \mathrm{T}, 1.57 \mu \mathrm{T}$, $1.96 \mu \mathrm{T}, 2.35 \mu \mathrm{T}, 2.74 \mu \mathrm{T}, 3.13 \mu \mathrm{T}, 3.52 \mu \mathrm{T}, 3.91 \mu \mathrm{T}$, and $4.31 \mu \mathrm{T})$ and similar sequence parameters to the ones used for QUEST measurements.

\section{$T_{1}$ and $T_{2}$ Measurements in vivo}

To calculate exchange rates using the standard methods and compare them with the PRO-QUEST sequence, $T_{1}$ and $T_{2}$ values are necessary (Equation 8). An inversion recovery EPI sequence was used to quantify the $T_{1}$ values [24]. A global adiabatic inversion pulse ( $\mathrm{fa}=180^{\circ}$, duration $=2 \mathrm{~ms}$ ) was applied followed by 10 inversion times exponentially spaced from $8.1 \mathrm{~ms}$ to $7.5 \mathrm{~s}$ (see Supporting Table S3). Other parameters were as follows: $T R=15 \mathrm{~s}$, TE $=25.5 \mathrm{~ms}$, slice thickness $=2 \mathrm{~mm}, \mathrm{FOV}=20 \times 20 \mathrm{~mm}^{2}$, matrix size $=64 \times 64$.

For the quantification of $T_{2}$ values, a CPMG sequence was used [25], consisting of a $90^{\circ}$ Sinc-shaped excitation pulse along the $x$ axis (duration $=2 \mathrm{~ms}$ ) followed by 15 Sincshaped refocusing pulses along the $y$ axis $\left(\mathrm{fa}=180^{\circ}\right.$, duration $\left.=1.6 \mathrm{~ms}\right)$. Other parameters were as follows: $T R=3 \mathrm{~s}$, TCPMG $=8.33 \mathrm{~ms}$, slice thickness $=2 \mathrm{~mm}, \mathrm{FOV}=20 \times 20 \mathrm{~mm}^{2}$, matrix size $=64 \times 64$.

\section{Post-Processing}

Data processing was performed using custom-written scripts in MATLAB (Mathworks Waltham, MA). All PRO-QUEST images were fitted following the steps described in the theory section. QUEST and QUESP data were analyzed following [10] by fitting full Zspectra obtained at 77 frequency offsets with different irradiation times or amplitudes respectively. $T_{1}$ and $T_{2}$ maps were obtained assuming mono-exponential decay for longitudinal and transverse relaxation [24],[25]. 
PRO-QUEST: A rapid method for CEST exchange rate quantification

\section{Results}

\section{Exchange rate measurements in samples using PRO-QUEST and QUEST}

Simulated data were obtained using a modified two-pool Bloch equations to assess the effect of different parameters such as $T_{1}$, exchange rates, magnetic field strength $\left(B_{0}\right)$, RF saturation power $\left(B_{1}\right)$ and finally the small flip angle $(\vartheta)$ on the saturation recovery curves in the presence of off-resonance RF irradiation (see Supporting Figures S2-S6).

Figure 2 shows the experimentally measured saturation recovery curves for PBS and $100 \mathrm{mM}$ alanine samples at different $\mathrm{pH}$ values with and without off-resonance progressive saturation pulses. The plots in Figure 2(a) demonstrate that saturation recovery curves obtained using a Look-Locker scheme in the absence of off-resonance CEST saturation pulses are not very sensitive to changes in $\mathrm{pH}$. However, changes in $\mathrm{pH}$ can be mapped for each sample when the same scheme is used with CEST saturation pulses applied on-resonance with the amine group of alanine (at 3.0ppm) (Figure 2(b)).

Quantitative estimates of exchange rates using both PRO-QUEST and QUEST, $T_{1}$ and $\mathrm{T}_{2}$ of PBS in the presence of alanine, glutamine, glutamate and taurine are displayed in Table 1. As can be seen, $T_{1}$ values exhibited no dependence on $\mathrm{pH}$ while $\mathrm{T}_{2}$ and exchange rates varied dramatically with $\mathrm{pH}$, thereby demonstrating that chemical exchange in alanine, glutamine, glutamate and taurine are base-catalyzed. Note that the exchange rates of the (protonated) amine groups in amino acids depend on $\mathrm{pH}, \mathrm{pKa}$ and the type and concentration of the buffer system used. The pKa values of the amino acids used in this study are: alanine 9.7, glutamate 9.5, glutamine 9.1, and taurine 9.0. If aqueous solutions were used, the expected exchange rates should be the fastest for taurine and slower for alanine. Because the samples were titrated in $0.1 \%$ PBS, the exchange rates are affected by additional reaction pathways.

It is worth noting that the PRO-QUEST exchange rates of glutamine and taurine at the highest $\mathrm{pH}$ values (7.4 and 7.2) decreased to $5.80 \times 10^{3} \mathrm{~s}^{-1}$ and $3.13 \times 10^{3} \mathrm{~s}^{-1}$ respectively. A reduction in the exchange rates of glutamine and taurine at $\mathrm{pH} 7.4$ and $\mathrm{pH} 7.2$ was also obtained when QUEST analysis was performed. These results suggest 
PRO-QUEST: A rapid method for CEST exchange rate quantification

that quantification of exchange rates using saturation times at the power used does not works for exchange rates above $\sim 15 \times 10^{3} \mathrm{~s}^{-1}$. This trend was not observed with QUESP analysis (see Supporting Table S1-S2 and Supporting Figures S7, S8) where the exchange rates in taurine and glutamine continued to increase with $\mathrm{pH}$ up to $20 \times 10^{3} \mathrm{~s}^{-1}$.

PRO-QUEST sensitivity to changes in the concentration of exchangeable protons was evaluated by increasing solute concentrations from $12.5 \mathrm{mM}$ to $100 \mathrm{mM}$ (Table 2). $\mathrm{T}_{1}$ did not change when the concentration of solute increased and was found to be equal to $2.95 \pm 0.01 \mathrm{~s}$ in all the samples. The exchange rates however were found to be equal within error for $12.5,25$ and $50 \mathrm{mM}$ in alanine and glutamine, but deviated for $100 \mathrm{mM}$. The exchange rates of glutamate were $0.77 \times 10^{3} \mathrm{~s}^{-1}$ on average. The measured exchange rates for taurine at high concentrations were found to be increased by one order of magnitude compared to the exchange rates measured at lower concentrations. In addition, the $T_{2}$ was found to vary with the solute concentration for all samples. All data were fitted by keeping the concentration $\rho_{B}$ to its theoretical value, calculated from the number of protons in the solute multiplied by the concentration of the agent divided by the concentration of protons in the solvent (111.2M), see Table 2, while fitting the saturation recovery curves at different irradiation amplitudes for the exchange rates.

Figure 3(a-d) displays the $\overline{R_{e x}^{\prime}}$ values obtained for each sample at different mean $\mathrm{B}_{1}$ values. In all experiments, $\overline{R_{e x}^{\prime}}$ increased with $B_{1}$ (Figures $3(a-d)$ ). Changes in the exchange rates of alanine, glutamine, glutamate and taurine with $\mathrm{pH}$ are shown in Figure 4(a). Figure 4(b) displays the measured exchange rates for all the samples when the solute concentration was increased from $12.5 \mathrm{mM}$ to $100 \mathrm{mM}$.

\section{T1 measurements and error analysis in healthy rats}

$\mathrm{T}_{1}$ maps from healthy rats were acquired using PRO-QUEST without the off-resonance CEST pulses at three imaging flip angles $\left(8^{\circ}, 15^{\circ}, 25^{\circ}\right)$. Additional $T_{1}$ maps were also collected using an inversion recovery spin echo EPI sequence for comparison (Figure 5). Figure $5(a-d)$ shows the $T_{1}$ values obtained with IR-EPI vs Look-Locker acquisition in 3 different regions in a rat brain. $T_{1}$ was significantly smaller $(p=0.003)$ for $25^{\circ}$ and 
PRO-QUEST: A rapid method for CEST exchange rate quantification

was not significantly different $(p=0.19)$ for $8^{\circ}$ when Look Locker acquisition was used comparative to IR-EPI in all ROIs.

\section{$B_{1}$ effects on the measured $T_{1}$ using saturation recovery Look-Locker sequence}

A $B_{1}$ map can be calculated by acquiring data at two or more flip angles [21],[26]. If we consider a 3D acquisition, a discrepancy in the flip angle will mainly be due to $B_{1}$ effects. However, for a 2D acquisition slice profile effects need to be considered as well. For shaped RF pulses such as Gaussian pulses, the effective flip angle depends on the slice profile, which is not an ideal square profile. Figure $6(a)$ shows a $B_{1}$ map obtained in a healthy rat which includes both $B_{1}$ variations and slice profile effects. The $B_{1}$ map, calculated with $\vartheta_{1}=8^{\circ}, \vartheta_{2}=15^{\circ}$ and $\vartheta_{3}=25^{\circ}$, is expressed as a fraction of the ideal flip angle and the mean $B_{1}$ in this slice was found to be equal to 0.68 of the prescribed one. Figure $6(\mathrm{~b})$ displays the slice profile effects in the case of a Gaussian RF pulse with $\vartheta=8^{\circ}, 15^{\circ}$ and $25^{\circ}$. Solid lines represent the ideal or nominal slice profile ( $2 \mathrm{~mm}$ slice thickness, $\vartheta=25^{\circ}, 15^{\circ}$ and $8^{\circ}$ ) and the dashed lines represent the achieved percentage of RF pulse, calculated by comparing the area under the Gaussian pulses to the area of an ideal pulse with a constant flip angle throughout the slice. The simulations show that an applied flip angle of $8^{\circ}$ is underestimated to $74 \%$ of its ideal value. No $T_{1}$ or $T_{2}$ decay was considered during the pulse for slice profile simulations using simple numerical integration of the Bloch equations.

\section{Relaxation exchange weighted $\left(\overline{R_{e x}^{\prime}}\right)$ maps in healthy and infarcted rats}

Figure 7 shows PRO-QUEST curves obtained in a rat brain at $24 \mathrm{~h}$ post stroke. The $\overline{R_{e x}^{\prime}}$ of normal tissue calculated over the three rats was $2.6 \times 10^{-2} \pm 3 \times 10^{-4} \mathrm{~s}^{-1}$ whereas, in the stroke lesion seen in the right side of the brain it dropped to $6.3 \times 10^{-3} \pm 3 \times 10^{-4} \mathrm{~s}^{-1}$. Figures $8(\mathrm{a})$ and (b) display the $\overline{R_{e x}^{\prime}}$ maps obtained in a healthy rat brain before and $24 \mathrm{~h}$ post stroke at $0.39 \mu \mathrm{T}$, respectively. The ratio of the $\overline{R^{\prime}}$ ex at $0.39 \mu \mathrm{T}$ and $0.65 \mu \mathrm{T}$ is displayed in Figures 8 (c) and (d) before and $24 \mathrm{~h}$ post stroke respectively. The concentration-free $\overline{R_{e x}^{\prime}}$ map is sensitive to changes in exchange rate and $\omega_{1}$ but not to the concentration of the CEST pool and therefore it can numerically be solved to probe changes in $k_{e x}$ in vivo. Note that the concentration-free $\overline{R_{e x}^{\prime}}$ was reduced both on the 
PRO-QUEST: A rapid method for CEST exchange rate quantification

contra- and ipsilateral side of the stroke in the grey matter, indicating an overall reduction in exchange rate following stroke. 
PRO-QUEST: A rapid method for CEST exchange rate quantification

\section{Discussion}

In recent years, much effort has been focused on the measurement of amide proton transfer (APT)-weighted signal and other CEST signals using several CEST metrics obtained by various fitting approaches [27], [28], [29]. Here, we have implemented a new pulse sequence, called PRO-QUEST, which enables quantification of exchange rates and therefore isolation of the CEST effect within a clinically applicable total scan time. This sequence samples multiple evenly spaced points along a $T_{1}$ recovery curve after a global saturation pulse while off-resonance pulses label protons that are constantly exchanging with water. To progressively follow the water signal during offresonance irradiation and recovery, a standard Look-Locker sequence has been modified. In addition, a complete set of equations has been derived that describe the recovery of the magnetization due to relaxation and chemical exchange following the Look-Locker sampling scheme.

We showed using both simulated and experimental data that the accuracy in estimating exchange rates is strongly affected by changes in $T_{1}$ and $\vartheta$. Therefore, these parameters had to be mapped from additional acquisitions without the use of offresonance saturation pulses and used as input parameters for calculating the exchange rates in Equations 2 and 8. In addition, since the effect of exchange to the water signal is very small, the main contribution remains the direct saturation effect on the water, which is a function of $R_{1}, R_{2}$ and $\omega_{1}$. Here, we show that the direct effect can be fitted as a separate parameter $\overline{R_{e f f}}$ and therefore the calculated exchange-dependent relaxation rate $\overline{R_{e x}^{\prime}}$ which describes the CEST effect is isolated from confounding factors (see Equation 6).

Based on Equation 8, exchange rates were calculated in samples containing exchangeable protons in different exchange regimes including slow-to-intermediate and fast exchange regimes. These experiments showed that PRO-QUEST is sensitive to $\mathrm{pH}$ alterations through changes in exchange rates. Furthermore, as exchange rates in taurine, alanine, glutamate and glutamine are base-catalyzed, their exchange rates increase with $\mathrm{pH}$ (see Figure $3(\mathrm{e})$ ). The range of our calculated exchange rates in 
PRO-QUEST: A rapid method for CEST exchange rate quantification

amine protons is in line with previously published results [26],[27] and they are consistent with the results of the QUEST analysis. However, one should keep in mind that the original QUEST approach requires the acquisition to reach steady state and the data to be obtained by repeating the same sequence with at least five different saturation times or pulse train lengths. PRO-QUEST alleviates this requirement through the acquisition of 300 images collected at 300 saturation times in $2.8 \mathrm{~min}$. This would have resulted in being able to only acquire 31 images for a single frequency offset using the same sequence in a QUEST experiment. Note also that the use of a saturationrecovery was governed by the need to clearly establish the starting state of the system, but it is not a limiting factor. Indeed, the use of inversion pulses could also be envisaged but might need a longer TR to ensure reaching a steady state. A recent work [32] on positive CEST contrast explored the possibility to use a $180^{\circ}$ pulse followed by CEST off-resonance saturation pulse without interleaved readout.

The exchange rate or the exchange-dependent relaxation term Rex represents an additional spectrally selective relaxation pathway to the relaxation of the water magnetization in the rotating frame as can be seen from Equations 3, 7 and 8 . Considering that the same functional group (amine) has been studied in small molecules such as amino acids and exchange kinetics was different based on the type of amino acid, the exchange rates in proteins (bigger molecules with smaller correlation times) will be substantially slower than what we detected here mainly because the interior backbone protons are shielded from solvent [33]. In addition, because of the inherent limitations of CEST techniques, we are unable to discriminate the origin of the signals at $3.00 \mathrm{ppm}$ in vivo. So the measured Rex will be equal to the sum of each contribution weighted by its concentration (Equation 6). For example, faster or more concentrated species will contribute more. Rex is also dependent on the experimental conditions (i.e. irradiation amplitude and duration) and on the method used to calculate it. Finally, in vivo, the chemical exchange is restricted by membranes, while prolonged $\mathrm{T}_{2}$ effects affect the accessibility to the water pool and the presence of phosphates affect the buffering of the system. MT and dipole-dipole interactions are also confounding factor since they occur simultaneously and cannot be distinguished from 
PRO-QUEST: A rapid method for CEST exchange rate quantification

chemical exchange in Z-spectra, although they are slower processes and $\mathrm{pH}$ insensitive [34].

Our study demonstrates that PRO-QUEST substantially enhances imaging specificity to ischemic acidification during stroke relative to standard APT-weighted imaging and, when combined with perfusion and diffusion weighted imaging, should permit refined prediction of the salvageable tissue within the penumbra. Because the range of exchange rates in vivo seems to be dominated by slow base-catalyzed rates corresponding to amide protons, tissue acidification is associated with a reduced amide exchange rate as seen in Figure 8. Note that tissue acidification is associated with cell death and tissue damage [35]. This is mainly due to the fact that $\mathrm{pH}$ alterations are associated with imbalance in glucose/oxygen delivery and consumption during stroke, leading to anaerobic metabolism and mitochondrial poisoning [36]. Therefore, exchange relaxation or $\mathrm{pH}$ maps can serve as important surrogate metabolic imaging biomarkers even within the first few hours after stroke. Our results demonstrating a lowering of the exchange rate in the infarcted regions are in good agreement with previous findings [29] [30],[37] . However, the additional decrease in $\overline{R_{e x}^{\prime}}$ observed on the contralateral side has not been reported previously using APT-weighted imaging [37]. This finding, if confirmed, could be important to understand the pathophysiology of stroke. The lack of appearance of this effect on APT-weighted imaging could be due to low signal-to-noise ratio of the latter method. This would demonstrate an improved sensitivity of PROQUEST to this effect as compared with standard APT-weighted imaging.

In a recent study, perturbation of longitudinal magnetization rate in the rotating frame was proposed to reduce the effects of non-chemical exchange-related parameters (i.e. direct water saturation) in the transient state when a continuous wave saturation pulse was used [38]. An exchange-dependent relaxation rate (SPACER) was derived from $\Delta \mathrm{R}_{1 \rho}=\left(R_{1 \rho}^{r e f}-R_{1 \rho}^{\text {lab }}\right)$ where $R_{1 \rho}^{r e f}=R_{1 \rho}(+\Delta \omega), \quad R_{1 \rho}^{\text {lab }}=R_{1 \rho}(-\Delta \omega)$ based on several approximations including a sufficiently small $\left|\Delta R_{1 \rho}\right|$ and a fast exchange rate approximation. Although that study showed promise for small $B_{1}$ amplitudes, the mapping of faster exchange species requires high $B_{1}[1]$ and therefore this method is likely to be limited to slow-to-intermediate exchange regimes. In contrast to SPACER, 
PRO-QUEST: A rapid method for CEST exchange rate quantification

PRO-QUEST can be used to map exchange rates in different exchange regimes by replacing the off-resonance saturation pulse with a spin-lock module applied on the water resonance for probing fast exchanging species. In addition, PRO-QUEST measurements can be obtained at different frequency offsets and irradiation amplitudes to capture the build-up of the signal due to chemical exchange and other confounding effects, such as magnetization transfer without the need of acquiring full Z-spectra like SPACER.

Another pulse sequence used to separate and quantify both slow and fast exchanging protons without the need to record full Z-spectra is the variable-delay multipulse (VDMP) method, consisting of a series of saturation pulses separated by an interpulse delay time $t_{\text {mix }}$ [39]. Similarly to PRO-QUEST, the VDMP sequence requires only one irradiation frequency, by applying an RF pulse of high bandwidth $(200-600 \mathrm{~Hz})$, and two images with different delay times: the first is used as a reference image and the second provides the exchange-weighted contrast. The difference between VDMP and the PROQUEST method is that the saturation recovery PRO-QUEST curves are based on a $T_{1}$ mechanism, while VDMP is based on $T_{2}$. In VDPM, the authors manipulate the interpulse delay to gain insight into different exchange properties, while here we vary the PRO-QUEST curves through different irradiation amplitudes to target different exchange regimes. Alternatively, PRO-QUEST has the ability (not shown) to be used with interleaved on-resonance spin lock modules to capture fast exchanging species.

It has been shown that the application of a gradient field simultaneously with the saturation pulse allows obtaining highly accelerated multi-sample Z-spectra. Similar with PRO-QUEST, by repetitively interleaving gradient-encoded saturation and an echo planar imaging $(\mathrm{EPI})$ readout, an incremental saturation time is achieved, which enables exchange rate quantification through a QUEST type of analysis [40]. Both PRO-QUEST and ultrafast CEST accelerate the acquisition by modifying standard pulse sequences and thus making both methods easily available to many potential users. 
PRO-QUEST: A rapid method for CEST exchange rate quantification

\section{Limitations}

There are a few limitations of our study. First, since only one irradiation frequency is used, this method will be susceptible to Bo shifts. However, in an attempt to tackle this issue we used high bandwidth saturation pulses $(1000 \mathrm{~Hz}$ for phantom studies and 400 $\mathrm{Hz}$ for the in vivo study) and carefully set the frequency offset to zero using a PRESS sequence. Another limitation is that, in our study, exchange rate measurements using QUEST or PRO-QUEST are unreliable beyond $15 \times 10^{3} \mathrm{~s}^{-1}$. This indicates that we would need higher irradiation powers to target fast exchange rates using QUEST or PROQUEST from the ones used here or to use on-resonance interleaved spin lock modules for increased sensitivity in this regime. It is worth noting that if the labelling efficiency is

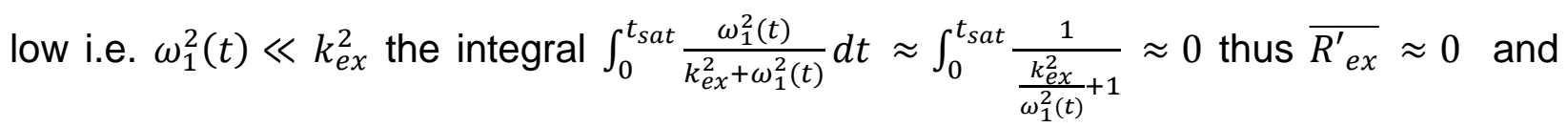
the PRO-QUEST curves will recover mainly with $R_{1}$ and $R_{2}$. An example of weak labelling is shown in Supporting Figure $\mathrm{S} 6$ in which the contribution of $\overline{R_{e x}^{\prime}}$ to the obtained PRO-QUEST curves is minimal for $k_{e x}>3000 s^{-1}$ when $\mathrm{B}_{1}=0.87 \mu \mathrm{T}$. 
PRO-QUEST: A rapid method for CEST exchange rate quantification

\section{Conclusion}

In this study, we developed and validated a new pulse sequence called PRO-QUEST by using full Bloch-McConnell simulations of a two pool system and by acquiring data both in vitro and in vivo. Based on simulations and experimental data, the calculated exchange rates were found to agree with the original QUEST method. The developed PRO-QUEST sequence offers a much faster alternative for calculating exchange rates and gaining insight into exchange processes in vivo. In particular, the application of PRO-QUEST in an animal model of stroke is encouraging as it shows its utility in a typical clinical application. 
PRO-QUEST: A rapid method for CEST exchange rate quantification

\section{Appendix}

Derivation of the water signal evolution as a function of saturation time with and without saturation pulses or spin lock modules

Consider $\mathrm{n}$ off-resonance saturation modules based on RF pulses or spin-lock modules of length tsat separated by an interval $\tau$-tsat as shown in Figure 1 . The evolution of the water signal as a function of the saturation time during the off-resonance saturation module can be written as:

$$
M_{z}\left(t_{s a t}\right)=M(t=0) e^{-R_{1} t_{s a t}}+M_{s s}\left(1-e^{-R_{1} t_{s a t}}\right)
$$

where $M(t=0)$ is the initial z-magnetization prior to the first saturation module. In the case of a saturation-recovery Look-Locker experiment $M(t=0)=M e q\left(1-e^{-\frac{t d}{T_{1}}}\right)$ where $t d$ is the time to first saturation pulse from the preparation. The normalized steady state signal is $\mathrm{M}_{\mathrm{ss}}=\frac{R_{1} \cos ^{2} \varphi}{R_{1 \rho}}$ where $\cos \varphi=1$ for spin-lock and $\cos \varphi=\frac{\Omega}{\sqrt{\omega_{1}^{2}+\Omega^{2}}}$ for CEST. $R_{1 \rho}$ is the longitudinal relaxation rate in the rotating frame. In the presence of offresonance irradiation, it is equal to the sum of the relaxation rates due to chemical exchange and water saturation.

After the off-resonance saturation module and similar to a typical Look-Locker experiment an RF pulse of small flip angle $(\vartheta)$ is used to sample multiple time points during the relaxation of the $z$-magnetization. The $R F(\vartheta)$ pulse is considered to cause

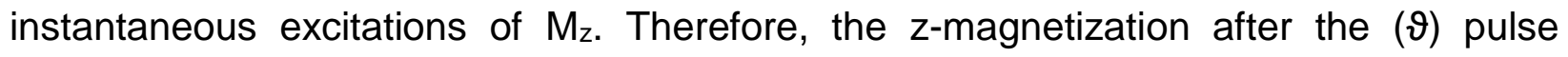
becomes $M_{z}\left(t_{s a t}\right) \cos \vartheta$.

\section{Solution without considering effects of exchange during the recovery between off- resonance saturation pulses}

Assuming a recovery of the $z$-magnetization during the $\tau$-tsat interval with the time constant $T_{1}$, i.e. without considering effects of exchange during the recovery between off-saturation pulses, the signal before the second saturation module can be expressed as: 
PRO-QUEST: A rapid method for CEST exchange rate quantification

$M_{z}\left(\tau-t_{s a t}\right)=M_{z}\left(t=t_{s a t}\right) e^{-\left(\tau-t_{s a t}\right) / T_{1}}+M e q\left(1-e^{-\left(\tau-t_{s a t}\right) / T_{1}}\right)$

Combining equations (A1) and (A2), $M_{z}$ can be written as:

$$
\begin{aligned}
& M_{z}(\tau)=\left[\operatorname{Meq}\left(1-e^{-\frac{t d}{T_{1}}}\right) e^{-R_{1} t_{s a t}}+M_{s s}\left(1-e^{-R_{1} t_{s a t}}\right)\right] \cos \vartheta e^{-\left(\tau-t_{s a t}\right) / T_{1}}+M e q(1- \\
& \left.e^{-\left(\tau-t_{\text {sat }}\right) / T_{1}}\right)
\end{aligned}
$$

To calculate the z-magnetization after the second off-resonance saturation module, equation (A3) is substituted into equation (A1) as the initial magnetization $M_{z}(t=0)=$ $M_{z}(t=\tau)$.

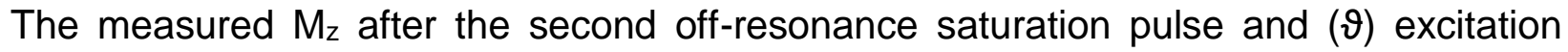
pulse is then:

$M_{z}(2 \tau)=\left[M e q\left(1-e^{-\frac{t d}{T_{1}}}\right) e^{-R_{1} t_{s a t}}+M_{S S}\left(1-e^{-R_{1} t_{s a t}}\right)\right][1+$

$\left.\cos \vartheta e^{-\left(\tau-t_{s a t}\right) / T_{1}} e^{-R_{1 \rho} t_{s a t}}\right]+\left[M e q\left(1-e^{-\frac{\tau-t_{s a t}}{T_{1}}}\right) e^{-2 R_{1} t_{s a t}}(\cos \vartheta)^{2} e^{-2 \frac{\left(\tau-t_{s a t}\right)}{T_{1}}}\right]$

Each iteration of the next off-resonance saturation followed by a Look-Locker read out multiplies the $\mathrm{M}_{\mathrm{z}}(\mathrm{n}-1)$ by a factor $\cos \vartheta e^{-\left(\tau-t_{s a t}\right) / T_{1}} e^{-R_{1 \rho} t_{s a t}}$ and adds Meq $(1-$ $\left.e^{-\frac{t d}{T_{1}}}\right) e^{-R_{1 \rho} t_{s a t}}+M_{s S}\left(1-e^{-R_{1} t_{s a t}}\right)$. Finally, the desired expression for $\mathrm{M}_{z}$ following a train of $\mathrm{n}$ off-resonance saturation pulses and $(\vartheta)$ excitation pulses is given by:

$$
\begin{aligned}
& M_{z s a t}(n \tau)=\frac{1-\left[\alpha(\cos \theta)^{n} e^{-n\left(\tau R_{1}-t_{s a t}\left(R_{1}-R_{1} \rho\right)\right)}\right]}{\left.1-\left[\alpha(\cos \theta) e^{-\left(\tau R_{1}-t_{s a t}\left(R_{1}-R_{1} \rho\right)\right.}\right)\right]} M_{z s a t}(\tau) \\
& \quad+M_{e q}\left(1-e^{-\left(t_{d} R_{1}\right)}\right)\left[(\cos \theta)^{n} e^{-n\left(\tau R_{1}-t_{s a t}\left(R_{1}-R_{1 \rho}\right)\right)}\right] \\
& \text { where } M_{\text {zsat }}(\tau)=M_{s s}\left(1-e^{-\left(R_{1} t_{s a t}\right)}\right) \cos \theta e^{-\left(\left(\tau-t_{s a t}\right) R_{1}\right)}+M_{\text {eq }}\left(1-e^{-\left(\left(\tau-t_{s a t}\right) R_{1}\right)}\right)
\end{aligned}
$$


PRO-QUEST: A rapid method for CEST exchange rate quantification

Let us consider equation (A1) again. Assuming recovery of the z-magnetization with $R_{1 A}$ and chemical exchange during the interpulse delay $t_{d}=\tau-t_{s a t}$, the signal before the second saturation pulse or spin-lock module can be expressed as [41],[42]:

$M_{z}\left(\tau-t_{s a t}\right)=d a a\left[M_{z}\left(t=t_{s a t}\right)-M_{0 z}\right]+d a b\left[\Psi M_{z}\left(t=t_{s a t}\right)-\rho_{B} M_{0 z}\right]+M_{0 z}$

Combining equations (A1) and (A6) the $M_{z}$ can be written as:

$M_{z}(t d)=d a a\left[M e q\left(1-e^{-\frac{t d}{T_{1}}}\right) \cos \vartheta e^{-R_{1} t_{s a t}}+M_{S S} \cos \vartheta\left(1-e^{-R_{1} t_{s a t}}\right)-M_{0 z}\right]+$

$d a b\left[\Psi M e q\left(1-e^{-\frac{t d}{T_{1}}}\right) \cos \vartheta e^{-R_{1 \rho} t_{s a t}}+\Psi M_{S S} \cos \vartheta\left(1-e^{-R_{1} t_{s a t}}\right)-\rho_{B} M_{0 z}\right]+M_{0 Z}=$

$d a a \cdot u+d a b . v+M_{0 z}$

where

$d a a=\frac{1}{R_{1 A}-k_{e x}-\rho_{B} k_{e x}-R_{1 B}}\left[\left(R_{1 B}-2 \rho_{B} k_{e x}-k_{e x}-R_{1 A}\right) \cdot e^{-\frac{(\tau-\text { tsat })}{T_{1}}}+\left(2 R_{1 A}+\right.\right.$

$\left.\left.\rho_{B} k_{e x}\right) \cdot e^{-(\tau-t s a t) \cdot\left(\rho_{B} k_{e x}+k_{e x}+R_{1 B}\right)}\right]$,

$d a b=\frac{k_{e x}}{R_{1 A}-\rho_{B} k_{e x}-k_{e x}-R_{1 B}}\left(e^{-\frac{(\tau-t s a t)}{T_{1}}}-\right.$

$\left.e^{-(\tau-t s a t) \cdot\left(\rho_{B} k_{e x}+k_{e x}+R_{1 B}\right)}\right), \Psi=\rho_{B}-\frac{R_{e x}}{k_{e x}}$.

(A8)

To calculate the z-magnetization after the second off-resonance saturation pulse or spin-lock module equation (A7) is substituted into equation (A1) as the initial magnetization $\mathrm{M}_{\mathrm{z}}(\mathrm{t}=0)=\mathrm{M}_{\mathrm{z}}(\mathrm{t}=\tau)$.

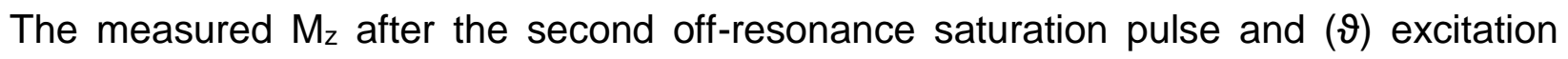
pulse is then:

$M_{z}(2 \tau)=(d a a+\Psi \cdot d a b)(d a a \cdot \mathrm{u}+d a b \cdot v) \cdot \cos \vartheta e^{-R_{1} t_{s a t}}+d a a \cdot x_{1}+d a b \cdot x_{2}+M_{0 z}(\mathbf{A} 9)$

where $x_{1}=M_{0 z} \cos \vartheta e^{-R_{1} t_{s a t}}+M_{S S} \cos \vartheta\left(1-e^{-R_{1 \rho} t_{s a t}}\right)-M_{0 z}$ and

$x_{2}=\Psi M_{0 Z} \cos \vartheta e^{-R_{1} t_{s a t}}+\Psi M_{S S} \cos \vartheta\left(1-e^{-R_{1 \rho} t_{s a t}}\right)-\rho_{B} M_{0 Z}$ 
PRO-QUEST: A rapid method for CEST exchange rate quantification

After the third module of CEST saturation pulse- $\vartheta$ pulse -delay the z-magnetization can be written as:

$M_{z}(3 \tau)=(d a a+\Psi \cdot d a b)^{2}($ daa.u + dab.v $) \cdot \cos ^{2} \vartheta e^{-2 R_{1 \rho} t_{s a t}}+[(d a a+$

$\left.\Psi \cdot d a b) \cdot \cos \vartheta e^{-R_{1 \rho} t_{s a t}}+1\right] \cdot\left(\right.$ daa. $x_{1}+$ dab. $\left.x_{2}\right)+M_{0 z}$

(A10)

If we set $\alpha=(d a a+\Psi \cdot d a b) \cdot \cos \vartheta e^{-R_{1 \rho} t_{s a t}}+1$ and expand using geometric series the desired expression for $\mathrm{M}_{z}$ following a train of $\mathrm{n}$ off-resonance saturation pulses and $(\vartheta)$ excitation pulses is given by:

$$
\begin{aligned}
& M_{z s a t}(n \tau)=\alpha^{n-1}\left[(d a a+\Psi \cdot d a b) \cdot \mathrm{u}+\operatorname{dab}\left(\Psi-\rho_{B}\right) M_{0 z}\right]+[(d a a+\Psi \cdot d a b) \cdot \mathrm{u}+ \\
& \left.(d a a+\Psi \cdot d a b)\left(M_{0 z} e^{-\frac{t d}{T_{1}}} \cdot e^{-R_{1 \rho} t_{s a t}}\right)+d a b\left(\Psi-\rho_{B}\right) M_{0 z}\right] \cdot \frac{1+\alpha-\alpha^{n-1}-\alpha^{n}}{1-\alpha^{2}}+M_{0 z}
\end{aligned}
$$

where

$d a a+\Psi \cdot d a b=\left(1-\frac{R_{e x}}{k_{e x}}\right) e^{-\frac{(\tau-t s a t)}{T_{1}}}$, and

$d a b=\frac{k_{e x}}{R_{1 A}-\rho_{B} k_{e x}-k_{e x}-R_{1 B}}\left(e^{-\frac{(\tau-t s a t)}{T_{1}}}-e^{-(\tau-t s a t) \cdot\left(\rho_{B} k_{e x}+k_{e x}+R_{1 B}\right)}\right)$

For data analysis, we used (A5) as described in the theory section. However, for completion of our theory we provide the solution (A11) when considering the effects of exchange during the recovery between off-resonance saturation pulses which will be crucial for slow exchange rates or for clinical systems where longer inter-pulse delays are used. In our study, the duty cycle was $88 \%$ and the samples used to validate PROQUEST possess exchangeable protons that resonate in the intermediate to fast exchange regime and therefore we assume that equation (A5) would be sufficient to 
PRO-QUEST: A rapid method for CEST exchange rate quantification

describe our data (i.e. $d a a+\Psi . d a b \approx e^{-\frac{(\tau-t s a t)}{T_{1}}}$ and $\left.d a b\left(\Psi-\rho_{B}\right) M_{0 z} \ll 1\right)$ in line with published studies [20],[39]. 
PRO-QUEST: A rapid method for CEST exchange rate quantification

\section{Acknowledgments}

The authors would like to thank Dr David Thomas, Aaron Kujawa, and Dr Mina Kim for careful reading of the manuscript. This project was funded by UCL Grand Challenge and supported by the National Institute for Health Research University College London Hospitals Biomedical Research Centre. 
PRO-QUEST: A rapid method for CEST exchange rate quantification

\section{Tables}

Table 1: Exchange rates and relaxation times $\left(T_{1}, T_{2}\right)$ in samples containing $100 \mathrm{mM}$ alanine, glutamine, glutamate and taurine at various $\mathrm{pH}$ values and at $23^{\circ} \mathrm{C}$

\begin{tabular}{|c|c|c|c|c|c|}
\hline \multirow{2}{*}{$\begin{array}{l}\text { Substance } \\
\text { Alanine }\end{array}$} & \multicolumn{5}{|c|}{$\mathrm{pH}$} \\
\hline & 6.02 & 6.33 & 6.74 & 7.06 & 7.42 \\
\hline $\mathrm{T} 1(\mathrm{~s})$ & $2.97 \pm 0.01$ & $2.95 \pm 0.01$ & $2.91 \pm 0.01$ & $2.94 \pm 0.01$ & $2.97 \pm 0.01$ \\
\hline $\mathrm{T} 2(\mathrm{~s})$ & $0.473 \pm 0.07$ & $0.295 \pm 0.07$ & $0.152 \pm 0.07$ & $0.096 \pm 0.07$ & $0.105 \pm 0.07$ \\
\hline \multicolumn{6}{|l|}{$k_{e x}\left(10^{3} s^{-1}\right)$} \\
\hline PRO-QUEST & $0.56 \pm 0.01$ & $0.91 \pm 0.06$ & $2.27 \pm 0.15$ & $6.47 \pm 1.06$ & $12.3 \pm 0.21$ \\
\hline \multicolumn{6}{|l|}{$k_{e x}\left(10^{3} s^{-1}\right)$} \\
\hline \multirow[t]{2}{*}{ QUEST } & $0.58 \pm 0.13$ & $0.99 \pm 0.05$ & $2.42 \pm 0.09$ & $7.67 \pm 3.51$ & $11.1 \pm 3.81$ \\
\hline & \multicolumn{5}{|c|}{$\mathrm{pH}$} \\
\hline Glutamate & 6.1 & 6.4 & 6.6 & 7.1 & 7.4 \\
\hline $\mathrm{T} 1(\mathrm{~s})$ & $2.71 \pm 0.01$ & $2.76 \pm 0.01$ & $2.77 \pm 0.01$ & $2.77 \pm 0.01$ & $2.81 \pm 0.01$ \\
\hline $\mathrm{T} 2(\mathrm{~s})$ & $0.429 \pm 0.04$ & $0.341 \pm 0.04$ & $0.359 \pm 0.04$ & $0.282 \pm 0.04$ & $0.222 \pm 0.04$ \\
\hline \multicolumn{6}{|c|}{ ים } \\
\hline PRO-QUEST & $0.71 \pm 0.03$ & $0.78 \pm 0.03$ & $0.84 \pm 0.03$ & $1.27 \pm 0.20$ & $2.29 \pm 0.15$ \\
\hline \multicolumn{6}{|l|}{$k_{e x}\left(10^{3} s^{-1}\right)$} \\
\hline \multirow[t]{2}{*}{ QUEST } & $0.72 \pm 0.22$ & $0.86 \pm 0.23$ & $1.04 \pm 0.19$ & $1.66 \pm 0.28$ & $2.54 \pm 0.34$ \\
\hline & \multicolumn{5}{|c|}{$\mathrm{pH}$} \\
\hline Glutamine & 6.1 & 6.4 & 6.6 & 7.1 & 7.4 \\
\hline $\mathrm{T} 1(\mathrm{~s})$ & $2.6 \pm 0.01$ & $2.6 \pm 0.01$ & $2.6 \pm 0.01$ & $2.6 \pm 0.01$ & $2.6 \pm 0.01$ \\
\hline $\mathrm{T} 2(\mathrm{~s})$ & $0.323 \pm 0.04$ & $0.180 \pm 0.04$ & $0.143 \pm 0.04$ & $0.112 \pm 0.04$ & $0.141 \pm 0.04$ \\
\hline \multicolumn{6}{|l|}{$k_{e x}\left(10^{3} s^{-1}\right)$} \\
\hline PRO-QUEST & $0.96 \pm 0.03$ & $1.85 \pm 0.03$ & $2.54 \pm 0.03$ & $12.6 \pm 0.20$ & $5.80 \pm 0.15$ \\
\hline \multicolumn{6}{|l|}{$k_{e x}\left(10^{3} s^{-1}\right)$} \\
\hline \multirow[t]{2}{*}{ QUEST } & $0.98 \pm 0.15$ & $1.90 \pm 0.09$ & $2.58 \pm 0.27$ & $12.6 \pm 0.26$ & $5.88 \pm 0.74$ \\
\hline & \multicolumn{5}{|c|}{$\mathrm{pH}$} \\
\hline Taurine & 5.85 & 6.08 & 6.31 & 6.86 & 7.2 \\
\hline $\mathrm{T} 1(\mathrm{~s})$ & $3.04 \pm 0.01$ & $3.05 \pm 0.01$ & $3.02 \pm 0.01$ & $3.08 \pm 0.01$ & $3.04 \pm 0.01$ \\
\hline $\mathrm{T} 2(\mathrm{~s})$ & $0.148 \pm 0.04$ & $0.097 \pm 0.04$ & $0.093 \pm 0.04$ & $0.131 \pm 0.04$ & $0.330 \pm 0.04$ \\
\hline \multicolumn{6}{|l|}{$k_{e x}\left(10^{3} \mathrm{~s}^{-1}\right)$} \\
\hline PRO-QUEST & $2.57 \pm 0.05$ & $3.27 \pm 0.87$ & $5.11 \pm 0.19$ & $16.0 \pm 0.16$ & $3.13 \pm 0.08$ \\
\hline \multicolumn{6}{|l|}{$k_{e x}\left(10^{3} \mathrm{~s}^{-1}\right)$} \\
\hline QUEST & $2.59 \pm 0.07$ & $5.27 \pm 0.33$ & $6.43 \pm 0.51$ & $14.5 \pm 0.51$ & $2.91 \pm 0.31$ \\
\hline
\end{tabular}


PRO-QUEST: A rapid method for CEST exchange rate quantification

Table 2: Exchange rates and relaxation times in samples containing alanine, glutamate, glutamine and taurine at various concentrations. The $\mathrm{pH}$ was constant at 6.2 and the temperature was $23^{\circ} \mathrm{C}$.

\begin{tabular}{|c|c|c|c|c|}
\hline \multirow{2}{*}{$\begin{array}{l}\text { Substance } \\
\text { Alanine }\end{array}$} & \multicolumn{4}{|c|}{ Concentration } \\
\hline & $12.5 \mathrm{mM}$ & $25 \mathrm{mM}$ & $50 \mathrm{mM}$ & $100 \mathrm{mM}$ \\
\hline $\mathrm{T} 1(\mathrm{~s})$ & $2.97 \pm 0.01$ & $2.95 \pm 0.01$ & $2.91 \pm 0.01$ & $2.94 \pm 0.01$ \\
\hline $\mathrm{T} 2(\mathrm{~s})$ & $0.90 \pm 0.14$ & $0.88 \pm 0.14$ & $0.62 \pm 0.14$ & $0.25 \pm 0.14$ \\
\hline \multicolumn{4}{|l|}{$k_{e x}\left(10^{3} \mathrm{~s}^{-1}\right)$} & $2.5 \times 10^{-3}$ \\
\hline \multirow[t]{2}{*}{ PRO-QUEST } & $0.70 \pm 0.46$ & $0.67 \pm 0.26$ & $0.74 \pm 0.10$ & $1.24 \pm 0.40$ \\
\hline & \multicolumn{4}{|c|}{ Concentration } \\
\hline Glutamate & $12.5 \mathrm{mM}$ & $25 \mathrm{mM}$ & $50 \mathrm{mM}$ & $100 \mathrm{mM}$ \\
\hline $\mathrm{T} 1(\mathrm{~s})$ & $2.94 \pm 0.01$ & $2.93 \pm 0.01$ & $2.92 \pm 0.01$ & $2.88 \pm 0.01$ \\
\hline $\mathrm{T} 2(\mathrm{~s})$ & $1.19 \pm 0.16$ & $8.90 \pm 0.16$ & $6.31 \pm 0.16$ & $3.36 \pm 0.16$ \\
\hline \multicolumn{5}{|l|}{$k_{e x}\left(10^{3} \mathrm{~s}^{-1}\right)$} \\
\hline \multirow[t]{2}{*}{ PRO-QUEST } & $0.71 \pm 0.31$ & $0.69 \pm 0.44$ & $0.82 \pm 0.16$ & $0.87 \pm 0.13$ \\
\hline & \multicolumn{4}{|c|}{ Concentration } \\
\hline Glutamine & $12.5 \mathrm{mM}$ & $25 \mathrm{mM}$ & $50 \mathrm{mM}$ & $100 \mathrm{mM}$ \\
\hline $\mathrm{T} 1(\mathrm{~s})$ & $2.96 \pm 0.01$ & $2.95 \pm 0.01$ & $2.94 \pm 0.01$ & $2.88 \pm 0.01$ \\
\hline $\mathrm{T} 2(\mathrm{~s})$ & $1.10 \pm 0.18$ & $0.82 \pm 0.18$ & $0.49 \pm 0.18$ & $0.19 \pm 0.18$ \\
\hline $\begin{array}{l}\rho_{\mathrm{B}} \\
k_{e x}\left(10^{3} \mathrm{~s}^{-1}\right)\end{array}$ & $7.8 \times 10^{-4}$ & $5.1 \times 10^{-4}$ & $1.2 \times 10^{-3}$ & $2.5 \times 10^{-3}$ \\
\hline \multirow[t]{2}{*}{ PRO-QUEST } & $0.64 \pm 0.58$ & $0.91 \pm 0.41$ & $1.10 \pm 0.16$ & $1.82 \pm 0.16$ \\
\hline & \multicolumn{4}{|c|}{ Concentration } \\
\hline Taurine & $12.5 \mathrm{mM}$ & $25 \mathrm{mM}$ & $50 \mathrm{mM}$ & $100 \mathrm{mM}$ \\
\hline $\mathrm{T} 1(\mathrm{~s})$ & $3.04 \pm 0.01$ & $3.05 \pm 0.01$ & $3.00 \pm 0.01$ & $2.96 \pm 0.01$ \\
\hline $\mathrm{T} 2(\mathrm{~s})$ & $0.92 \pm 0.16$ & $0.53 \pm 0.16$ & $0.21 \pm 0.16$ & $0.92 \pm 0.16$ \\
\hline $\begin{array}{l}\rho_{\mathrm{B}} \\
k_{e x}\left(10^{3} \mathrm{~s}^{-1}\right)\end{array}$ & $7.8 \times 10^{-4}$ & $5.1 \times 10^{-4}$ & $1.2 \times 10^{-3}$ & $2.5 \times 10^{-3}$ \\
\hline PRO-QUEST & $0.99 \pm 0.58$ & $1.88 \pm 0.35$ & $3.29 \pm 0.65$ & $5.93 \pm 0.97$ \\
\hline
\end{tabular}


PRO-QUEST: A rapid method for CEST exchange rate quantification

\section{Figure legends:}

Figure 1 PRO-QUEST Pulse sequence diagram. An initial $90{ }^{\circ}$ saturation pulse(s) was followed by off-resonance saturation pulses or spin-lock modules (a) or by delays (b) interleaved with the acquisition of segmented exchange-weighted images. Following the application of a $90^{\circ}$ pulse, Ny lines in k-space are acquired by the application of the same number of slice-selective $\vartheta$ pulses. After full relaxation, a new $90^{\circ}$ pulse is applied and the following $\mathrm{Ny}$ lines in k-space are acquired in an identical manner. This is repeated until the whole of k-space, at multiple time-points, has been acquired. The sequence in $1 b)$ is used for $T_{1}$ measurement and the resulting parameters $\left(T_{1}\right.$, and $\left.B_{1}\right)$ are then used as input parameters for fitting the results from the PRO-QUEST sequence in 1 (a) to obtain the exchange-related relaxation rate.

Figure 2 Experimental saturation recovery curves without (a) and with (b) the application of saturation pulses in samples consisting of $100 \mathrm{mM}$ of Alanine at $\mathrm{pH}=6.02$, $6.33,6.74,7.06$ and 7.42. (c) Fitted and experimental data for Alanine at $\mathrm{pH}=7.0$ are shown. The fitted curve was obtained by fitting the experimental data to equation 2. (d) Theoretical curves of Alanine at $\mathrm{pH}=6.02$ without and with off-resonance saturation pulses obtained using Equations 1 and 2 using the values of the measured parameters reported in Table 1.

Figure 3 (a-d) Measured $\overline{R_{e x}^{\prime}}$ values using equations (2) and (8) in alanine (Ala), glutamine (Gln), glutamate (Glu) and taurine (Tau) samples respectively for $B_{1}$ values ranging from $0.87 \mu \mathrm{T}$ to $8.67 \mu \mathrm{T}$. The calculated exchange rates for each sample are shown in Table 1.

Figure 4 (a) and (b) display alterations in exchange rates of alanine, glutamine, glutamate and taurine due to changes in $\mathrm{pH}$ and concentration respectively.

Figure 5 (a-d) $T_{1}$ values from three representative regions in a healthy rat obtained using a saturation-recovery Look-Locker sequence at $\vartheta=8^{\circ}, 15^{\circ}, 25^{\circ}$ and IR-EPI sequence. (e) $T_{1}$ map obtained in a healthy rat brain using an Inversion recovery spinecho EPI sequence. 
PRO-QUEST: A rapid method for CEST exchange rate quantification

Figure 6 (a) $B_{1}$ map obtained from a healthy rat with $\vartheta_{1}=8^{\circ}, \vartheta_{2}=15^{\circ}$ and $\vartheta_{3}=25^{\circ}$ (b) slice profile effects for Gaussian RF pulses. The solid lines show the ideal slice profile ( $2 \mathrm{~mm}$ slice thickness, $\vartheta=8^{\circ}, 15^{\circ}$ and $25^{\circ}$ ) and the dashed lines show the achieved flip angles calculated as the area under the Gaussian-shaped profiles as a percentage of the area of an ideal pulse with constant flip angle throughout the slice.

Figure 7(a) A single pixel chosen in the stroke lesion (red) and contralateral area (blue) overlaid on a Diffusion Weighted Image (DWI), (b) experimental data and fitted PROQUEST curves from a pixel in the stroke (red) and contralateral region (blue).

Figure $8 \overline{R_{e x}^{\prime}}$ maps in a healthy rat brain (a) and at $24 \mathrm{~h}$ post stroke (b) at irradiation amplitude of $0.39 \mu \mathrm{T}$. The concentration-free $\overline{R_{e x}^{\prime}}=\overline{R_{e x}^{\prime}}(0.39 \mu \mathrm{T}) / \overline{R_{e x}^{\prime}}(0.65 \mu \mathrm{T})$ is shown in 8 (c) and 8 (d) for healthy and infarcted rat respectively. 
PRO-QUEST: A rapid method for CEST exchange rate quantification

\section{References}

1. van Zijl PCM, Yadav NN. Chemical exchange saturation transfer (CEST): what is in a name and what isn't? Magn Reson Med. 2011 Apr;65(4):927-48.

2. McMahon MT, Gilad AA, Zhou J, Sun PZ, Bulte JWM, van Zijl PCM. Quantifying exchange rates in chemical exchange saturation transfer agents using the saturation time and saturation power dependencies of the magnetization transfer effect on the magnetic resonance imaging signal (QUEST and QUESP): Ph calibration for poly-Llysine and a starburst dendrimer. Magn Reson Med. 2006 Apr;55(4):836-47.

3. Liepinsh E, Otting G. Proton exchange rates from amino acid side chains-implications for image contrast. Magn Reson Med. 1996 Jan;35(1):30-42.

4. Zhou J, Lal B, Wilson DA, Laterra J, van Zijl PCM. Amide proton transfer (APT) contrast for imaging of brain tumors. Magn Reson Med. 2003 Dec;50(6):1120-6.

5. Sun PZ, Zhou J, Sun W, Huang J, van Zijl PC. Detection of the Ischemic Penumbra Using pH-Weighted MRI. J Cereb Blood Flow Metab. 2007 Jun 1;27(6):1129-36.

6. Mori S, Abeygunawardana C, van Zijl PC, Berg JM. Water exchange filter with improved sensitivity (WEX II) to study solvent-exchangeable protons. Application to the consensus zinc finger peptide CP-1. J Magn Reson B. 1996 Jan;110(1):96-101.

7. Dixon WT, Ren J, Lubag AJM, Ratnakar J, Vinogradov E, Hancu I, et al. A concentration-independent method to measure exchange rates in PARACEST agents. Magn Reson Med. 2010 Mar;63(3):625-32.

8. van Zijl PCM, Zhou J, Mori N, Payen J-F, Wilson D, Mori S. Mechanism of magnetization transfer during on-resonance water saturation. A new approach to detect mobile proteins, peptides, and lipids. Magn Reson Med. 2003 Mar;49(3):440-9.

9. Mori S, Berg JM, van Zijl PC. Separation of intramolecular NOE and exchange peaks in water exchange spectroscopy using spin-echo filters. J Biomol NMR. 1996 Jan;7(1):77-82. 
PRO-QUEST: A rapid method for CEST exchange rate quantification

10. Zaiss M, Angelovski G, Demetriou E, McMahon MT, Golay X, Scheffler K. QUESP and QUEST revisited - fast and accurate quantitative CEST experiments. Magn Reson Med. : doi:10.1002/mrm.26813.

11. Zaiss $M$, Bachert $P$. Exchange-dependent relaxation in the rotating frame for slow and intermediate exchange - modeling off-resonant spin-lock and chemical exchange saturation transfer. NMR Biomed. 2013 May 1;26(5):507-18.

12. Sun PZ. Simplified quantification of labile proton concentration-weighted chemical exchange rate ( $\mathrm{k}(\mathrm{ws})$ ) with RF saturation time dependent ratiometric analysis (QUESTRA): normalization of relaxation and RF irradiation spillover effects for improved quantitative chemical exchange saturation transfer (CEST) MRI. Magn Reson Med. 2012 Apr;67(4):936-42.

13. Magnetic Resonance Fingerprinting - a promising new approach to obtain standardized imaging biomarkers from MRI. Insights Imaging. 2015 Mar 24;6(2):163-5.

14. Huang S, Cohen O, McMahon T.M, Kim R. Y, Rosen M, and Farrar C, "Quantitative chemical exchange saturation transfer imaging with magnetic resonance fingerprinting (MRF) ", In Proceedings of the 26 ${ }^{\text {th }}$ Annual Meeting of ISMRM, Hawaii, USA, 2017.

15. Zhengwei $\mathrm{Z}$, Pei $\mathrm{H}$, and Debiao L, "CEST Fingerprinting: A novel approach for exchange rate quantification", In Proceedings of the $26^{\text {th }}$ Annual Meeting of ISMRM, Hawaii, USA, 2017.

16. Look DC, Locker DR. Time Saving in Measurement of NMR and EPR Relaxation Times. Rev Sci Instrum. 1970 Feb 1;41(2):250-1.

17. Deichmann R, Haase A. Quantification of T1 values by SNAPSHOT-FLASH NMR imaging. J Magn Reson 1969. 1992 Feb 15;96(3):608-12.

18. Kay I, Henkelman RM. Practical implementation and optimization of one-shot T1 imaging. Magn Reson Med. 1991 Dec;22(2):414-24. 
PRO-QUEST: A rapid method for CEST exchange rate quantification

19. Jin T, Autio J, Obata T, Kim S-G. Spin-locking vs. chemical exchange saturation transfer MRI for investigating chemical exchange process between water and labile metabolite protons. Magn Reson Med. 2011 May;65(5):1448-60.

20. Meissner J-E, Goerke S, Rerich E, Klika KD, Radbruch A, Ladd ME, et al. Quantitative pulsed CEST-MRI using $\Omega$-plots. NMR Biomed. 2015 Oct 1;28(10):1196208.

21. Parker DL, Christian BA, Goodrich KC, Alexander AL, Buswell HR, Yoon C "Improved accuracy in T1 measurements". In Proceedings of the $6^{\text {th }}$ Annual Meeting of ISMRM, Sydney, Australia, 1998 p.2172.

22. Kiozumi J, Yoshida Y, Nakazawa T, Ooneda G. "Experimental studies of ischemic brain edema: I: A new experimental model of cerebral embolism in rats in which recirculation can be introduced in the ischemic area ". Japanese J Stroke. 1986; 8:1-8.

23. Tannús A. Adiabatic pulses. NMR Biomed. 1997;10(8):423-34.

24. Gowland PA, Stevenson VL. T1: The Longitudinal Relaxation Time. In: Tofts P, editor. Quantitative MRI of the Brain. John Wiley \& Sons, Ltd; 2003. p. 111-141.

25. Meiboom S, Gill D. Modified Spin-Echo Method for Measuring Nuclear Relaxation Times. Rev Sci Instrum. 1958 Aug 1;29(8):688-91.

26. Shin W, Gu H, Yang Y. Fast High-Resolution T1 Mapping using InversionRecovery Look-Locker Echo-Planar Imaging at Steady State: Optimization for Accuracy and Reliability. Magn Reson Med Off J Soc Magn Reson Med Soc Magn Reson Med. 2009 Apr;61(4):899-906.

27. Chappell MA, Donahue MJ, Tee YK, Khrapitchev AA, Sibson NR, Jezzard P, et al. Quantitative Bayesian model-based analysis of amide proton transfer MRI. Magn Reson Med. 2013 Aug 1;70(2):556-67.

28. Zhou IY, Wang E, Cheung JS, Zhang X, Fulci G, Sun PZ. Quantitative chemical exchange saturation transfer (CEST) MRI of glioma using Image Downsampling Expedited Adaptive Least-squares (IDEAL) fitting. Sci Rep. 2017 Dec;7(1):84. 
PRO-QUEST: A rapid method for CEST exchange rate quantification

29. Zaiss M, Xu J, Goerke S, Khan IS, Singer RJ, Gore JC, et al. Inverse Z-spectrum analysis for spillover-, MT-, and T1 -corrected steady-state pulsed CEST-MRI-application to pH-weighted MRI of acute stroke. NMR Biomed. 2014 Mar;27(3):240-52.

30. Jin T, Wang P, Zong X, Kim S-G. Magnetic resonance imaging of the AmineProton EXchange (APEX) dependent contrast. Neurolmage. 2012 Jan 16;59(2):121827.

31. Cai K, Haris M, Singh A, Kogan F, Greenberg JH, Hariharan H, et al. Magnetic resonance imaging of glutamate. Nat Med. 2012 Feb;18(2):302-6.

32. Vinogradov E, Soesbe TC, Balschi JA, Sherry AD, Lenkinski RE. pCEST: Positive Contrast Using Chemical Exchange Saturation Transfer. J Magn Reson. 2012 Feb;215:64-73.

33. Mäkelä HI, Gröhn $\mathrm{OHJ}$, Kettunen MI, Kauppinen RA. Proton Exchange as a Relaxation Mechanism for T1 in the Rotating Frame in Native and Immobilized Protein Solutions. Biochem Biophys Res Commun. 2001 Dec 14;289(4):813-8.

34. Jin $\mathrm{T}$, Wang $\mathrm{P}$, Zong $\mathrm{X}$, Kim S-G. MR imaging of the Amide-Proton Transfer effect and the pH-insensitive Nuclear Overhauser Effect at 9.4 T. Magn Reson Med Off J Soc Magn Reson Med Soc Magn Reson Med. 2013 Mar 1;69(3):760-70.

35. Zhou J, Payen J-F, Wilson DA, Traystman RJ, Van Zijl PCM. Using the amide proton signals of intracellular proteins and peptides to detect $\mathrm{pH}$ effects in MRI. Nat Med. 2003;9(8):1085-90.

36. Bainbridge A, Tachtsidis I, Faulkner SD, Price D, Zhu T, Baer E, et al. Brain mitochondrial oxidative metabolism during and after cerebral hypoxia-ischemia studied by simultaneous phosphorus magnetic-resonance and broadband near-infrared spectroscopy. Neurolmage. 2014 Nov 15;102 Pt 1:173-83.

37. Sun PZ, Murata Y, Lu J, Wang X, Lo EH, Sorensen AG. Relaxationcompensated fast multislice amide proton transfer (APT) imaging of acute ischemic stroke. Magn Reson Med. 2008 May;59(5):1175-82. 
PRO-QUEST: A rapid method for CEST exchange rate quantification

38. Wang $\mathrm{Y}$, Zhang $\mathrm{Y}$, Zhao $\mathrm{X}, \mathrm{Wu} \mathrm{B}$, Gao J-H. Perturbation of longitudinal relaxation rate in rotating frame (PLRF) analysis for quantification of chemical exchange saturation transfer signal in a transient state. Magn Reson Med. 2016 Nov 1;.

39. Xu J, Yadav NN, Bar-Shir A, Jones CK, Chan KWY, Zhang J, et al. Variable delay multi-pulse train for fast chemical exchange saturation transfer and relayednuclear overhauser enhancement MRI. Magn Reson Med. 2014 May;71(5):1798-812.

40. Xiang X, Yadav N. N, Song X, McMahon T. M, Jerschow A, van Zijl P.M.C, Xu J Screening CEST Contrast Agents Using Ultrafast CEST Imaging. 2016, J. Magn. Reson, in press, http://dx.doi.org/10.1016/j.jmr.2016.02.015

41. Roeloffs V, Meyer C, Bachert P, Zaiss M. Towards quantification of pulsed spinlock and CEST at clinical MR scanners: an analytical interleaved saturationrelaxation (ISAR) approach. NMR Biomed. 2015 Jan 1;28(1):40-53.

42. Zhou J, Wilson DA, Sun PZ, Klaus JA, Van Zijl PCM. Quantitative description of proton exchange processes between water and endogenous and exogenous agents for WEX, CEST, and APT experiments. Magn Reson Med. 2004 May;51(5):945-52. 
PRO-QUEST: A rapid method for CEST exchange rate quantification

\section{Supplemetary data:}

Supporting Figure S1: Z-spectra obtained at $0.78 \mu \mathrm{T}$ from $100 \mathrm{mM}$ of glutamine, taurine, glutamate and alanine.

Supporting Figure S2: Simulated 2-pool PRO-QUEST curves at different $T_{1}$. The simulation indicates the sensitivity of the PRO-QUEST measurements to changes in $T_{1}$. For all the simulations $\mathrm{B}_{0}=9.4 \mathrm{~T}, \vartheta=8^{\circ}, \mathrm{k}_{\mathrm{sw}}=1000 \mathrm{~Hz}, \mathrm{~B}_{1}=0.87 \mu \mathrm{T}$.

Supporting Figure S3: displays PRO-QUEST curves obtained by simulating a two-pool system at different $B_{0}$. For all the simulations $T_{1}=2.9 \mathrm{~s}, \vartheta=8^{\circ}, \mathrm{k}_{\mathrm{sw}}=1000 \mathrm{~Hz}, \mathrm{~B}_{1}=$ $0.87 \mu \mathrm{T}$.

Supporting Figure $\mathbf{S} 4$ displays simulated data obtained at various tip angles $\vartheta$. For all the simulations $\mathrm{T}_{1}=2.9 \mathrm{~s}, \mathrm{~B}_{0}=9.4 \mathrm{~T}, \mathrm{~B}_{1}=0.87 \mu \mathrm{T}, \mathrm{k}_{\mathrm{sw}}=1000 \mathrm{~Hz}$.

Supporting Figure $\mathbf{S} 5$ shows simulated data obtained at different irradiation powers $B_{1}$. For all the simulations $\mathrm{T}_{1}=2.9 \mathrm{sec}, \vartheta=8^{\circ}, \mathrm{ksw}_{\mathrm{sw}}=1000 \mathrm{~Hz}, \mathrm{~B}_{0}=9.4 \mathrm{~T}$.

Supporting Figure S6: Simulated two pool saturation recovery curves obtained at various exchange rates. For all the simulations $\mathrm{T}_{1}=2.9 \mathrm{sec}, \vartheta=8^{\circ}, \mathrm{B}_{0}=9.4 \mathrm{~T}, \mathrm{~B}_{1}=$ $0.87 \mu \mathrm{T}$.

Supporting Figure S7: Z-spectra obtained from taurine at (a) $\mathrm{pH}=5.85$, (b) $\mathrm{pH}=6.08$ and (c) $\mathrm{pH}=6.31$.

Supporting Figure S8: Z-spectra obtained from taurine at (a) $\mathrm{pH}=6.86$, (b) $\mathrm{pH}=7.2$ and (c) PBS.

Supporting Table S1: Exchange rates in alanine, glutamine, glutamate and taurine at various $\mathrm{pH}$ values

SupportingTable S2: Exchange rates in alanine, glutamine, glutamate and taurine at various concentrations

Supporting Table S3: inversion times $\mathrm{TI}$ for calculation of $\mathrm{T}_{1}$ used in phantoms and in vivo 
PRO-QUEST: A rapid method for CEST exchange rate quantification 